Paul Chorin, Florian Moreau, Didier Saury, Heat transfer modification induced by a localized thermal disturbance in a differentially-heated cavity, International Journal of Thermal Sciences, Volume 125, March 2018, Pages 101-110, ISSN 1290-0729, https://doi.org/10.1016/j.ijthermalsci.2017.11.018 (https:/www.sciencedirect.com/science/article/pii/ S129007291730563X)

\title{
Heat transfer modification induced by a localized thermal disturbance in a differentially-heated cavity
}

\author{
Paul Chorin, Florian Moreau*, Didier Saury* \\ Institut Pprime, UPR CNRS 3346, CNRS - ENSMA - Université de Poitiers, Département FTC, Téléport 2, 1 avenue \\ Clément Ader, BP40109, F-86961 Futuroscope Cedex, France \\ * corresponding authors. Tel.: +33 549498100 Email addresses: didier.saury@ensma.fr (D. Saury), florian.moreau@ensma.fr (F. Moreau).
}

\begin{abstract}
Numerical investigation of a natural convection flow with a localized thermal disturbance is presented here. The configuration of a differentially heated cavity is considered with air as working fluid. In this study, the initial flow regime is steady and close to the transition to unsteadiness. Different cases of local wall temperature disturbances are tested in a thin area at the onset of the hot vertical boundary layer. These cases are distinguished by the time-averaged temperature of this area (higher or lower than the hot wall temperature) and by their temporal evolution (steady or periodic). In cases with a periodic disturbance, the imposed frequency corresponds to the first frequency emerging in the unsteady regime. The results show the spread of temperature fluctuations across the whole cavity with the periodic disturbance, in particular near the upper corner of the cavity. The hot steady disturbance trigs the onset of time-dependent flow. Moreover, the local modification of the hot wall temperature has an impact on global heat transfer: about $16 \%$ downstream the disturbance area and about $2 \%$ on the opposite cold wall.
\end{abstract}

Keywords: Natural convection, Heat transfer modification, Differentially-heated cavity, Unsteady flows 


\section{Nomenclature}

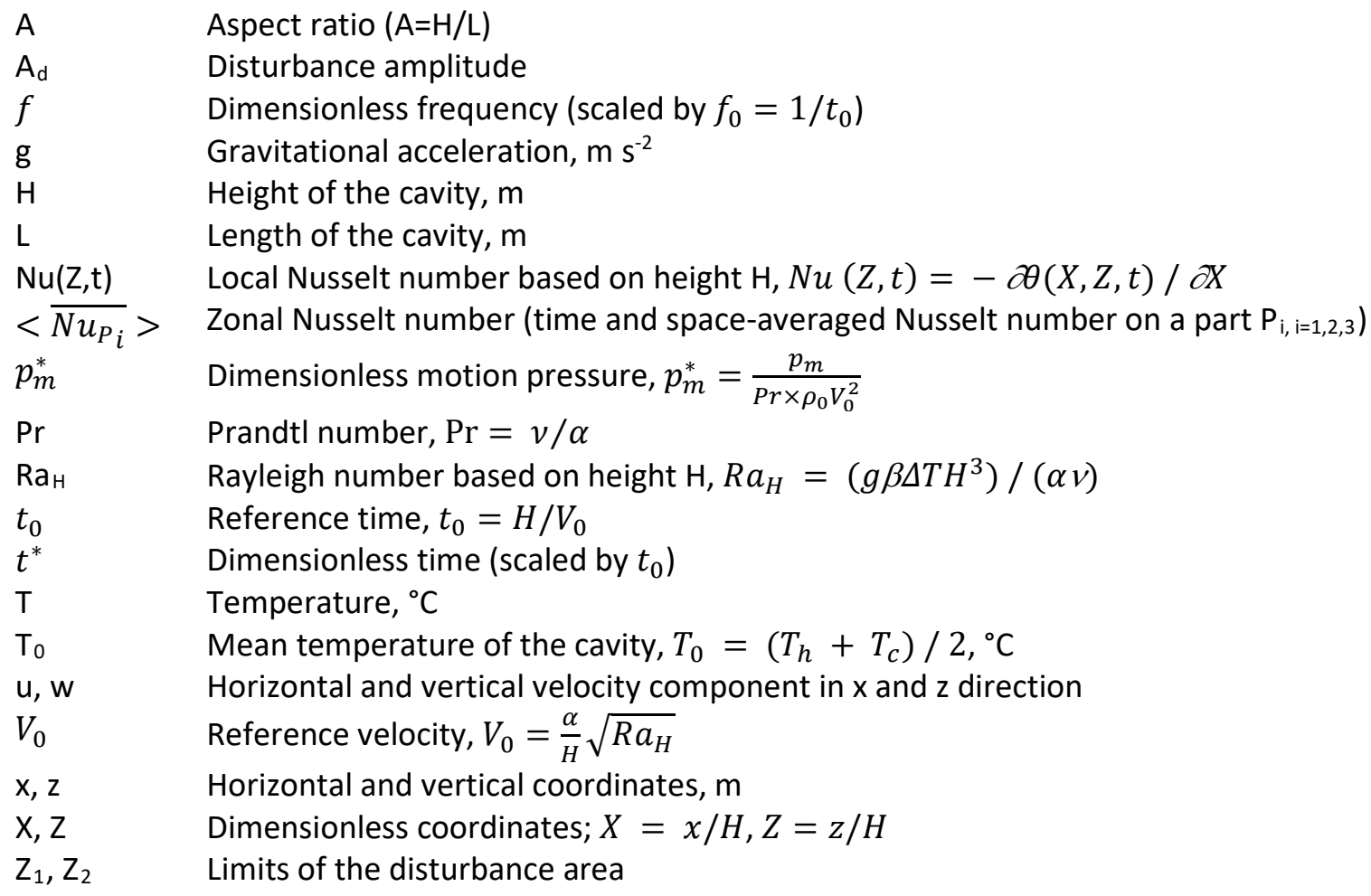

Greek symbols

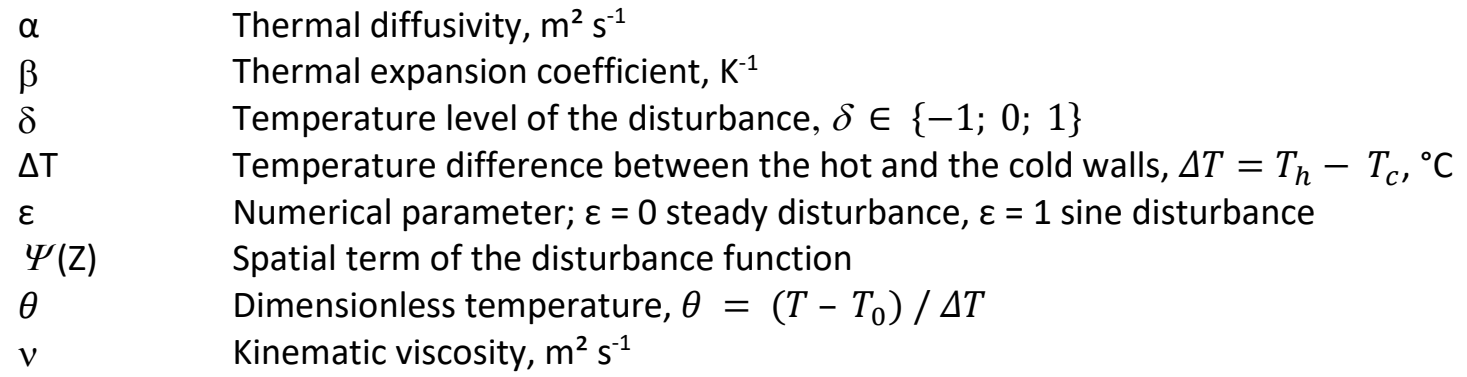

\section{Subscripts and Superscripts}

$\begin{array}{ll}\mathrm{c} & \text { Cold wall } \\ \mathrm{h} & \text { Hot wall } \\ \mathrm{d} & \text { Disturbance } \\ \mathrm{rms} & \text { Root mean square } \\ \mathrm{crit} & \text { critical } \\ 0 & \text { Reference value } \\ \Phi^{\prime} & \text { Fluctuation of } \Phi, \Phi^{\prime}=\Phi-<\Phi> \\ \bar{\Phi}(\mathrm{t}) & \text { Spatial averaged value of } \mathrm{h}, \bar{\Phi}(t)=\int_{0}^{1}<\Phi(Z, t)>d Z \\ <\Phi(\mathrm{Z})> & \text { Time average value of } \mathrm{h},<\Phi(Z)>=f_{d} \int_{t}^{t+1 / f_{d}} \Phi(Z, t) d t \\ * & \text { Dimensionless quantity } \\ - & \text { Steady disturbance } \\ \sim & \text { Sine disturbance }\end{array}$




$\begin{array}{ll}\text { CS } & \text { Cold Spot } \\ \text { HS } & \text { Hot Spot } \\ \text { DHC } & \text { Differentially Heated Cavity }\end{array}$

\section{Introduction}

Natural convection flows occur in a wide range of practical applications in which heat transfer optimization is of great interest: a better insulation of the walls such as in buildings construction, enhancement of thermal flux for cooling electronic device or nuclear reactors, as for thermal storage tanks and solar energy collectors.

These practical applications can be modeled through the simpler representation of the differentially heated cavity (DHC) in order to have a better understanding of the associated physics. DHC have been deeply studied experimentally and numerically for decades since the pioneering works of Batchelor [1]. In 1983 de Vahl Davis and Jones [2] published a benchmark solution in an air-filled square DHC for a Rayleigh number varying in the range of $10^{3}-10^{6}$. In the next years, several studies have been made to investigate the transition from a steady to a time-dependent flow [3-6]. Tric et al. [7] proposed 3D accurate solutions of air natural convection in a cubic DHC by mean of pseudo-spectral Chebyshev algorithm. Xin and Le Quéré [8-11] revisited the onset of time-dependent flows for aspect ratios between 1 and 8, for both adiabatic and perfectly conducting horizontal walls. They used several stability analysis algorithms to provide accurate critical Rayleigh numbers and associated frequencies of the most unstable modes.

Several authors have used multiple strategies to act on buoyancy-driven flows. For a heated vertical plate with a superimposed perturbation source, Zhao et al. [12] observed numerically a net heat transfer enhancement by a resonance-induced advancement of the laminarturbulent transition. For the Rayleigh-Bénard convection, Howle [13-14] investigated the control of the flow using localized heat fluxes, whereas Abourida et al. [15] and Douamna 
et al. [16] chose to impose a time-dependent temperature at the isothermal walls. By varying amplitude, period and dephasing between walls, heat transfer was changed. Hossain and Floryan investigated the control of natural convection in a fluid layer heated from below [17] or from below and above [18] with a sine spatial distribution. They found a significant increase of heat transfer when a synchronized disturbance is applied over both walls. Focusing now on the DHC configuration, one of the first ways to act on the flow in DHC was by means of a thin fin on the hot wall, positioned horizontally [19-22] or tilted [23,24], and more recently by using a flexible fin [25]. As buoyancy-driven flows can be induced by means of appropriate temperature boundary conditions, several studies have been carried out on the thermal disturbance in DHC instead of a mechanical one. In 1996, Kwak et al. [26,27] imposed numerically a sine-varying temperature on the hot wall of a square cavity for a fixed Rayleigh number and varied the amplitude and the frequency. The wall temperature oscillation caused an increase in time-average heat transfer, which was maximal at the resonant frequency. More recently, Mahapatra et al. [28,29] used alternatively active heat sources on the bottom wall of an enclosure. Heat transfer was higher compared to the case with a single steady heater and increased with frequency. In an experimental study, Penot et al. [30] investigated the effect of a thin pipe localized close to the hot wall. The pipe temperature was varied periodically at the resonant frequency of the critical flow regime. The thermal disturbances increased velocity fluctuations, but the introduction of the pipe acted in the opposite manner due to an obstacle effect, and a decrease of the global Nusselt number is observed.

In this paper, a thermal disturbance localized on the hot wall is used as actuator to act on natural convection and to modify heat transfer. Wall temperature is modified on a thin area at the onset of the hot vertical boundary layer. An aspect-ratio of 4 is considered, being the first aspect-ratio for which unsteady flow displays traveling waves near the cavity walls [10]. 
Air is taken as working fluid. In such a case, the flow is steady laminar up to the critical Rayleigh number of $10^{8}$ where unsteady regime appears in the outer edge of the boundarylayers. A 2D numerical study is performed in order to evaluate the feasibility and the pertinence of such an approach. The flow is idealized with a bi-dimensional aspect, since the first weak 3D flow does not alter the transition scenario to unsteadiness [11].

In the following sections, the model, the numerical methods and the validation are provided. Then an analysis of the results in terms of temperature fluctuations in the whole cavity and in terms of heat transfer through the active walls is carried out.

\section{Model and validation}

The DHC considered in this study is a parallelepiped-shaped cavity of aspect ratio $H / L=4$. It is composed of two vertical opposite isothermal walls, whereas the others walls are adiabatic (see Fig. 1). Two different temperatures $\left(T_{c}\right.$ and $\left.T_{h}\right)$ are imposed on these isothermal walls. Air thermophysical properties are evaluated at the mean temperature of the cavity $T_{0}=$ $\frac{T_{c}+T_{h}}{2}=20^{\circ} \mathrm{C}$. Due to small temperature variation in the cavity (the temperature difference between active walls is at worse $\Delta T=T_{h}-T_{C}=2.7^{\circ} \mathrm{C}$ ), these properties are given constant in the computational domain, except for the density in the buoyant term where the Boussinesq approximation is used. The characteristic Rayleigh number is $R a_{H}=9.0 \times 10^{7}$, close to the critical value. In this study, the physical lengths are scaled

by $\mathrm{H}$ (see Fig. 1): $(X, Z)=\left(\frac{x}{H}, \frac{Z}{H}\right)$. The dimensionless temperatures and velocities are defined by $\theta=\frac{T-T_{0}}{\Delta T}$ and $\mathbf{V}=\left(\frac{u}{V_{0}}, \frac{w}{V_{0}}\right)$, where $V_{0}=\frac{\alpha}{H} \sqrt{R a_{H}}$ denotes the reference velocity. The frequencies are scaled by using the characteristic time $t_{0}=\frac{H}{V_{0}}=\frac{H^{2}}{\alpha \sqrt{R a_{H}}}$. The dynamic of the system as well as the heat transfer are governed by the dimensionless continuity, momentum and energy conservation equations: 


$$
\begin{gathered}
\boldsymbol{\nabla} \cdot \boldsymbol{V}=0 \\
\frac{\partial \boldsymbol{V}}{\partial t^{*}}+\boldsymbol{V} \cdot \boldsymbol{\nabla} \boldsymbol{V}=-\boldsymbol{\nabla} p_{m}^{*}+\operatorname{Pr} \theta \boldsymbol{e}_{\boldsymbol{z}} \frac{P r}{\sqrt{R a_{H}}} \nabla^{2} \boldsymbol{V} \\
\frac{\partial \theta}{\partial t^{*}}+\boldsymbol{V} \cdot \boldsymbol{\nabla} \theta=\frac{1}{\sqrt{R a_{H}}} \nabla^{2} \theta
\end{gathered}
$$

Fig. 1 shows the scheme of the studied cavity and gathers the boundary conditions used. The vertical walls have isothermal boundary conditions with $\theta_{h}=0.5$ on the left wall and $\theta_{c}=$ -0.5 on the right wall, whereas horizontal walls are adiabatic: $\frac{\partial \theta}{\partial Z}(Z=0)=0$ and $\frac{\partial \theta}{\partial Z}(Z=1)=0$. For all walls, no slip boundary condition is applied: $U=W=0$.

Numerical study of 2D DHC is carried out by means of a co-localized finite-volume approach [31]. The spatial derivatives are discretized using second-order schemes for the temperature and the velocity, whereas integration in time is performed with a second order Crank-Nicholson scheme. The nodes of the grid are spread under a hyperbolic cosine distribution function in each direction $(\cosh (4 A X-2) ; \cosh (4 Z-2))$ to obtain a well refine mesh near the walls. To ensure that the results obtained do no depend on the number of grid elements, a grid independency test is performed. This test conducted on grids 60x120, 100x200, 130x260 and 160x320 confirms that the grid $130 \times 260$ is the most suitable grid to obtain accurate solutions with small CPU times. This grid will be used in the present study. The time step is chosen as the maximal value to keep the CFL number lower than 1.

The evolution of the global (time- and space-averaged) Nusselt number versus Rayleigh number both on a logarithmic scale is plotted in Fig. 2. The corresponding Rayleigh number varies between $5 \times 10^{7}$ and $1.9 \times 10^{8}$. We find the law $<\overline{N u}>=(0.0741 \pm 0.0006) R a_{H}^{0.252 \pm 0.0005}$, very close to the correlation from Grondin [32] for a DHC with aspect ratio $4:\langle\overline{N u}\rangle=$ $0.0725 R a_{H}^{0.25}$ 
The transition to time-dependent flow is conducted by successive Hopf bifurcations between which the mode frequencies of the travelling waves are stable at the first order [10]. The Rayleigh numbers of these bifurcations are defined as critical Rayleigh numbers. By varying the Rayleigh number from $9 \times 10^{7}$ to $1.8 \times 10^{8}$ without thermal disturbance, five timedependent branches of solutions for the unsteady Navier-Stokes equations are found. The corresponding frequencies and critical Rayleigh numbers are presented in Table 1. Frequencies $\mathrm{f}_{\text {crit }}$ are determined at the first Rayleigh number after the bifurcation by a Fourier analysis, whereas critical Rayleigh number are calculated by extrapolating the square signal amplitude (at point M, see Fig. 1) to zero, which is characteristic of a Hopf bifurcation. These results are in very good agreement with the benchmark solutions from [10]. A maximum deviation of $2 \%$ is found on the critical Rayleigh of the first branch. These results on heat transfer and unsteady behavior validate the numerical methods.

According to the results of Gadoin et al. [33], the optimal location to disturb the flow is at the beginning of the boundary layers, where the boundary layer modes have the greatest receptivity. Hence, in the cases with disturbance, the boundary temperature is modified on the hot wall $(X=0)$ between elevations $Z_{1}=0.20$ and $Z_{2}=0.25$, called the disturbance area. This disturbance area is localized outside the circulating region downstream the corner, and enables to obtain a large area downstream along the hot wall, where the effect on heat transfer will be analyzed. The temperature law applied in the disturbance area $\left(Z \in\left[Z_{1} ; Z_{2}\right]\right)$ is written in equation (4), where spatial term $\Psi(Z)$ is given in equation (5).

$$
\begin{gathered}
\theta_{d}(Z, t)=\theta_{h}+A_{d}\left(\delta+\varepsilon \sin \left(2 \pi f_{d} \frac{t}{t_{0}}\right)\right) \times \Psi(Z) \\
\Psi(Z)=\frac{1}{2}\left[1+\cos \left(2 \pi \frac{Z-Z_{\text {cent }}}{Z_{2}-Z_{1}}\right)\right]
\end{gathered}
$$


where $\mathrm{A}_{\mathrm{d}}$ is the imposed amplitude, $\delta \in\{-1 ; 0 ; 1\}$ denotes the mean temperature level of the disturbance (respectively below, at the same level or above $\theta_{h}$ ), $\varepsilon \in\{0 ; 1\}$ enables to choose between a steady disturbance $(\varepsilon=0)$ and a sine disturbance $(\varepsilon=1)$, and $\mathrm{f}_{\mathrm{d}}$ is the imposed disturbance frequency. Table 2 gathers investigated cases. The spatial term $\Psi(Z)$ given in

equation (5) is centered on the middle of the disturbance area $Z_{\text {cent }}=\frac{Z_{1}+Z_{2}}{2}$ and spatially smooths the disturbance to avoid discontinuities and be more realistic from a practical point of view. Three parts $\left(P_{1}, P_{2}, P_{3}\right)$ are defined respectively upstream the disturbance area $\left(P_{1}\right)$, downstream the disturbance area $\left(P_{2}\right)$ and all along the cold wall $\left(P_{3}\right)$ for further investigations (see Fig. 1). The temporal evolution of the imposed wall temperature at the center of the disturbance area, $\theta_{d}\left(Z=Z_{\text {cent }}\right)$, is plotted in Fig. 3 for all cases.

\section{Thermal perturbation and results analysis}

The effects of the disturbance on the flow are analyzed in terms of temperature fluctuations and heat transfer changes. For all these studies, the Rayleigh number is imposed at $9.000 \times 10^{7}$, slightly below the first critical Rayleigh number $R a_{H c}=1.052 \times 10^{8}$ (see Table 1 ): without any disturbance the flow is steady. All studies presented here below are carried out with an amplitude $A_{d}=1$ for cases with disturbance and $A_{d}=0$ for the reference case (ref. case).

\subsection{Influence on the temperature and the associated flow regime}

As a first way to illustrate the disturbance effect and its spread downstream the disturbance area, time-series of temperature evolution at a measuring point $\mathrm{M}$ (located close to the outer edge of the thermal boundary layer in $\mathrm{X}_{\mathrm{M}}=0.0375$ and $\mathrm{Z}_{\mathrm{M}}=0.9$, see Fig. 1) are plotted in Fig. 4. Note that the boundary layer thickness is defined as the location where, on the horizontal thermal profile, the temperature is equal to the core temperature at the considered elevation. 
Without disturbance (ref. case), the temperature is steady due to a sub-critical Rayleigh number and its value is $\theta_{M}=0.2569$. For a cold steady disturbance at $\theta_{d}(Z)=\theta_{h}-\Psi(Z)$ (case CS-), a slight decrease of $\theta_{M}$ is noticed (1.2\%), but no fluctuations are detected. With the cold sine disturbance (case $\mathrm{CS} \sim$ ), the change in mean value of temperature at point $\mathrm{M}\left\langle\theta_{M}>\right.$ is more important, around $6 \%$ lower than its value in ref. case. Fluctuations are observed with a frequency equal to the disturbance frequency $f_{M}=f_{d}=0.40$, and the corresponding amplitude is equal to $6 \%$ of the imposed amplitude. Note that this periodic evolution observed in Fig. 4 results from a distortion of the initial sine shape of the disturbance (see Fig. 3). This distortion expresses the presence of harmonics of this frequency. For a hot steady disturbance at $\theta_{d}(Z)=\theta_{h}+\Psi(Z)$ (case HS-), the mean temperature at point $\mathrm{M}$ has slightly increased $(+1.5 \%)$. Moreover, the flow does not remain steady and exhibits periodic fluctuations at $f_{M}=$ 0.408: $\theta_{M, r m s}=0.002$. This frequency and the associated amplitude level correspond to a non-perturbed cavity with a Rayleigh number slightly higher than the first critical Rayleigh number. Thus the onset of time-dependent flow is trigged by a thin hot-spot placed on the hot wall with a Hopf bifurcation, even without any unsteadiness introduced. In case HS (hot sine disturbance), the temperature exhibits larger amplitude of fluctuations when compared to case HS-, since a rms-value of $\theta_{M, r m s}=0.033$ is achieved, 15 times higher than the value obtained for HS- case. Moreover, fluctuations occur at the frequency of the disturbance: $f_{M}=f_{d}$. Thus even at an elevation $Z_{M}=0.9$ (0.65 above the end of the disturbance area), the induced fluctuations are still higher than $5 \%$ of their imposed value $\left(\theta_{d, r m s}=0.707\right)$. To conclude, whatever the disturbance, at this location, the mean temperature is modified (consequences in terms of heat transfer will be analyzed later on). However, only periodic disturbance (hot or cold) or steady hot disturbance trig an unsteady behavior.

In order to check if these temperature fluctuations exists all over the cavity as well as their spatial distribution, 2D fields of rms-temperature, $\theta_{r m s}$, are drawn in Fig. 5 . In the ref. case, the 
sub-critical regime is considered thus no temperature fluctuation is detected. These observations remain true in case CS-, and are consistent with the results obtained at the point $\mathrm{M}$ where no variation is noticed (see Fig. 4). On the contrary, fluctuations exist in case CS and reach $2 \times 10^{-3}$ upstream the disturbance area (close to $P_{1}$ ) and $5 \times 10^{-2}$ downstream (close to $P_{2}$ ). In this case, the fluctuations are observed in the whole cavity, which is also true for the case HS . However, in HS and CS cases, their local amplitude is not equally distributed. Several orders of magnitude between the different regions are observed: $10^{-1}-10^{-2}$ in the hot boundary layer versus $10^{-3}-10^{-4}$ in the core region. Concerning the case HS-, even if the disturbance is steady, fluctuations are only observed outside the core region, at the top and bottom parts of the cavity. An unsteady regime with temperature fluctuations located near the cavity walls is observed, typically encountered in cavity with aspect ratio $H / L$ higher or equal to 4 [10]. The flow presents unsteady laminar boundary layers with Tollmien-Schlichting waves travelling along their outer edges. The maximal temperature fluctuation is $\theta_{r m s}^{H S-}=4.5 \times 10^{-3}$ at the position $(X=0.025 ; Z=0.909)$, relatively close to the hot wall. The fluctuations have almost the same amplitude on the cold wall, although no disturbance at this side has been set. The propagation of the temperature disturbance in cases HS- and HS is plotted in Fig.6 in terms of instantaneous temperature fluctuations, $\theta^{\prime}=\theta-\langle\theta\rangle$. The six fields cover one temporal period. Temperature fluctuations in the case HS- are mainly present at the end of the boundary layer, and are in the order of magnitude of $10^{-3}$. This temperature field is close to those obtained for supercritical flow regimes [10] except that the temperature fluctuations are not centro-symmetrical here. Indeed, they are higher at the end of the hot boundary layer due to the location of the disturbance area with respect to the direction of the flow. In the case HS , temperature fluctuations $\left(\theta^{\prime}\right)$ gain one order of magnitude compared to the corresponding steady case (HS-). Hot and cold structures observed in this figure are defined respectively as areas where $\theta^{\prime}$ is positive or negative. In case HS , the structure number is increased when compared 
to case HS-. These structures are no more only located at the end of the boundary layers but all along the walls. These structures are then advected by the mean flow. For example, in the HS case, the hot structure which starts to climb in the second image $\left(t=t_{0}+1 / 6 f_{d}\right)$ close to the disturbance area. At time $t=t_{0}+5 / 6 f_{d}$, this hot structure starts to coalesce with another hot structure and form larger structure that can be observed $(Z=0.7)$ on the first image. Each structure can be tracked similarly. Moreover, these structures move faster downstream the disturbance area than at the end of the boundary layer. That is why these structures are more stretched in this area. This figure also highlights that the temperature fields are affected by the disturbance due to the existence of advected structures whose effects strongly depend on the type of the disturbance (hot or cold, steady or sine). An analyze of the impact of the temperature field modifications on the wall heat transfer will be carried out in the next section.

\subsection{Impacts on wall heat transfer}

Heat transfers through the isothermal walls are quantified by the Nusselt number. The comparison of zonal Nusselt number $<\overline{N u_{P_{i}}}>$ (time- and space-averaged on the parts $P_{1}, P_{2}$ and $P_{3}$, see Fig. 1) at $R a_{H}=9.0 \times 10^{7}$ and for the different cases is shown in Table 3. In cases with a disturbance temperature lower than the wall temperature $\left(\theta_{d}(Z, t)<\theta_{h}\right.$, cases CS- and CS ), an increase of the zonal Nusselt number on $P_{1}$ and $P_{2}$ is observed. This increase is small on $P_{1}$, less than $0.9 \%$, and high on $P_{2}$, by $15.8 \%$. On the contrary, when the disturbance temperature is higher than the wall temperature $\left(\theta_{d}(Z, t)>\theta_{h}\right.$, cases HS- and HS $)$, a reduction of $<\overline{N u_{P_{1}}}>$ and $<\overline{N u_{P_{2}}}>$ is observed with approximatively the same level in the two cases, less than $2 \%$ on $P_{1}$, and around $17 \%$ on $P_{2}$. Moreover, a sine disturbance has no distinct effect on global heat transfer compared to the steady disturbance on all parts. However, on $\mathrm{P}_{3}$ the modification of the zonal Nusselt number acts in an opposite manner. Indeed, $<\overline{N u_{P_{3}}}>$ decreases for the cold disturbances, and increases for the hot disturbances. These 
values are smaller than on $P_{2}$, close to $2.2 \%$. Thus, the temperature change near the disturbance area is spread along the whole cavity: the colder temperature downstream the disturbance area in cases CS- and CS increases the heat transfer downstream the disturbance, but decreases it on the cold wall. These results are reversed for cases HS- and HS .

\subsubsection{Spatial-variation of local time-averaged wall heat transfer}

In order to understand the spatial modification of the heat transfer on parts $P_{1}, P_{2}$ and $P_{3}$ due to the disturbance, the variation of the local Nusselt with the vertical position $\mathrm{Z}$ is plotted in Fig. 7 for the hot wall and in Fig. 8 for the cold wall. These local Nusselt numbers $<N u_{h}(Z)>$ and $<N u_{c}(Z)>$ are averaged in time over one time period of the disturbance. To highlight the effect, a superimposed graph shows the relative gains or losses in local heat transfer with respect to the ref. case, $\% N u_{Z}=\frac{\left.<N u_{Z}>-N u_{Z} \text { (ref.case }\right)}{N u_{Z}(\text { ref.case })}$, for part $\mathrm{P}_{1}$ in Fig. 7 and part $\mathrm{P}_{3}$ in Fig. 8. Downstream the disturbance area $\left(\mathrm{P}_{2}\right.$ part), the local Nusselt number is strongly reduced for the hot disturbances and increased for the cold disturbances, due to the change of the temperature field in the flow (Fig. 9). $<N u_{h}(Z)>$ gradually returns to the value obtained in the ref. case. Upstream the disturbance ( $\mathrm{P}_{1}$ part), a slight change is noticed compared to ref. case, which never exceed 2.4\% (HS case). These deviations are in accordance with the zonal values on $\mathrm{P}_{1}$ in Table 3 and are due to the change on the temperature of the return flow from $\mathrm{P}_{3}$ part (see Fig. 9).

Similarly, for $P_{3}$ part, the local Nusselt numbers are overall higher in cases HS- and HS compared to ref. case, and lower in cases CS- and CS $\sim$, which is in agreement with results given in Table 3. Although no significant difference is observed between zonal heat transfer for a steady and a sine disturbance (cases CS- and CS on one side, cases HS- and HS at the other side, see Table 3), their spatial distribution are substantially different (see Fig. 8). In the cases with a sine disturbance (dotted lines), a minimum value of $\% \mathrm{Nu}_{\mathrm{Z}}$ is reached for $Z \approx 0.9$, 
before an increase downstream. From $Z=0.7$ to the bottom of the cavity, \%Nuz averaged over this area is higher by $11 \%$ for the sine disturbances than for the corresponding steady ones.

These differences between the local values of $\left\langle N u_{c}(Z)\right\rangle$ are due to a difference on temperature distribution between the steady and the sine disturbances. In order to evaluate the local temperature modification induced by the disturbance, $<\theta_{C S-}-\theta_{\text {ref. case }}>$ and $<\theta_{C S \sim}-$ $\theta_{\text {ref. case }}>$ in the whole cavity are drawn in Fig. 9, completed with 4 snapshots of $\left(\theta_{C S \sim}-\right.$ $\theta_{\text {ref. case }}$ ) to observe its time-evolution. Streamlines are also overprinted (see movie of this time-evolution during one period is available with the online version of this article). In these CS- and CS cases, although the temperature is globally reduced, areas with higher temperature than in ref. case appear. In order to explain the higher value of \% $\mathrm{Nu}_{\mathrm{Z}}$ for $Z<0.7$ in the case CS compared to the case CS-, a focus on the core for $Z \approx 0.7$ is required (Fig. 9). A major difference between cases CS- and CS is observed at this elevation. Indeed, a hotter area exists in case CS . In this area, a part of the flow goes from the left (hot) to the right (cold) side. This air flow feeds the cold boundary layer, which increases the temperature difference and consequently $\% \mathrm{Nu}_{z}$ at this elevation for case CS . Moreover, snapshots of $\theta_{C S \sim}-\theta_{\text {ref. case }}$ (Fig. 9, right part) show that the heat transfer from the left to the right of the cavity is unsteady. This unsteady behavior is highlighted for $Z \in[0.4 ; 0.6]$, where the direction of the secondary flow in the core region changes on each snapshot. These complex unsteady phenomena and temperature differences in the core region cause the differences observed on the heat transfer distributions on $\mathrm{P}_{3}$ part for steady and sine disturbances, although the time-averaged values of zonal heat transfer remain very close.

To evaluate the impact of unsteady phenomena on local heat transfer, the time-averaged Nusselt number $<\mathrm{Nu}(\mathrm{Z})>$ along $P_{2}$ for the cases with a disturbance is shown in Fig. $10 .<\mathrm{Nu}(\mathrm{Z})>$ is framed by the local minimal and maximal values (dotted lines). In case CS-, the minimal and maximal values are equal to the time-averaged value since no fluctuations on $N u(Z, t)$ are 
detected. In case HS-, very weak fluctuations exist close to the upper corner, as observed in Fig. 6 for the temperature fluctuations. On the contrary, in the cases CS and HS the minimal and maximal values are substantially distinguished from the time-averaged values, and the two corresponding curves exhibits the envelope for the local Nusselt number. The difference between a cold disturbance and a hot one affects this envelope mainly in the region directly downstream the disturbance area, because of the strong modification of the time-averaged heat transfer occurring here (see Fig. 7). Farther from the disturbance area (for $\mathrm{Z}>0.60$ ), these two envelopes tend to reach the same shape whatever the case (HS or CS ).

\subsubsection{Time-evolution of local wall heat transfer}

As the time-averaged heat transfers are very close between the steady and sine disturbances, a focus on instantaneous wall heat transfer is carried out to well understand these similarities. Fig. 11 shows the evolution of local Nusselt number at the position $Z=0.3$ on the hot wall. This location (directly downstream the disturbance area) allows to observe the disturbance effect. $N u(Z=0.3)$ is obviously steady in ref. case since a sub-critical flow regime is achieved. In case CS- it remains steady, whereas in case CS oscillations are observed with a peak-to-peak amplitude around 3.4. For both cases the mean values are very close, with $0.6 \%$ of deviation. However, these mean values have increased by $27 \%$ when compared to the value of ref. case. For the hot disturbances cases (HS- and HS ), the mean values are very close as well and $29 \%$ below the value obtain for the ref. case. Therefore, the local heat transfer oscillates for the sine disturbances downstream the disturbance area, around the value for steady disturbances. This explains why no significant deviation is observed on time-averaged heat transfer between steady and sine disturbances. 
Moreover, it has been shown that the dynamic of the disturbances are responsible to the development of periodically hot and cold structures. In order to evaluate the impact of these structures on the instantaneous heat transfer, the profiles of local Nusselt number at different times for case HS on $P_{2}$ are shown in Fig. 12. The different times are equally distributed over a disturbance period. These profiles are consistent with the shape of the envelope plotted in Fig. 10. The snapshots of $N u(Z, t)$ are non-monotonic: they show important spatial oscillations caused by alternated hot and cold structures, which could not be highlighted with the envelope. Therefore, the sine disturbance creates strong oscillations on $N u(Z, t)$, in a similar manner to the propagation to a transversal wave with a fixed node in $Z=1$.

\section{Conclusion}

Numerical study of natural convection in differentially heated cavity with aspect ratio 4 has been performed with a localized thermal disturbance on the lower part of the hot wall. Four kinds of thermal disturbance have been tested according to the disturbance temperature (above or below the wall temperature) and its temporal evolution (steady or sinusoidal). Results have been analyzed in terms of temperature variations and heat transfer impact.

Except for the steady cold case (CS-), the thermal disturbance spreads through the cavity. The steady hot disturbance (HS- case) trigs the onset of a time-dependent supercritical flow, where fluctuations are located at the end of the vertical boundary layers and the horizontal wall jets. For the sine disturbances (CS and HS cases), temperature fluctuations reach the core region but remain much higher in the main flow. Alternating hot and cold structures are observed in the vertical boundary layers.

The wall heat transfer is affected spatially and temporally by the disturbances. The global heat transfer is increased by a hot disturbance and decrease by a cold disturbance, with a change around $2 \%$. Downstream the disturbance, the heat transfer is inversely modified, with a major 
change around $17 \%$. For the sine disturbances, the heat transfer oscillates around a value close to the value obtained for the steady disturbances. Thus no heat transfer enhancement is noticed by the introduction of periodic disturbance. On the cold wall, the heat transfer distribution is complex, due to local fluid regions with higher or lower temperature (when compared to the reference case) combined with the unsteady secondary flow coming from the hot wall.

This study indicates how the wall heat transfer in internal natural convection flows can be optimized with the modification on a small area of the wall thermal condition. Thus, a comparison of these results with the actual situation in an experimental DHC could be subject of future studies.

\section{References}

[1] G. K. Batchelor, "Heat transfer by free Convection Across a Closed Cavity between Vertical Boundaries at Different Temperatures», Q. Appl. Math., pp. 209-223, 1954.

[2] G. De Vahl Davis, I. P. Jones, "Natural convection in a square cavity: A comparison exercise», Int. J. Numer. Meth. Fluids, vol. 3, pp. 227-248, 1983.

[3] S. Paolucci, D. R. Chenoweth, "Transition to chaos in a differentially heated vertical cavity", J. Fluid Mech, vol. 201, pp. 379-410, 1989.

[4] M. R. Ravi, R. A. W. M. Henkes, C. J. Hoogendoorn, «On the high-Rayleigh-number structure of steady laminar natural-convection flow in a square enclosure», J. Fluid Mech, vol. 262, pp. 325351, 1994.

[5] R. J. A. Janssen, R. A. W. M. Henkes, «Influence of prandtl number on instability mechanisms and transition in a differentially heated square cavity» J. Fluid Mech, vol. 290, pp. 319-344, 1995.

[6] M. A. Christon, P. M. Gresho, S. B. Sutton, «Computational predictability of time-dependent natural convection flows in enclosures (including a benchmark solution) ", Int. J. Numer. Meth. Fluids, vol. 40, pp. 953-980, 2002.

[7] E. Tric, G. Labrosse, M. Betrouni, «A first incursion into the 3D structure of natural convection of air in a differentially heated cubic cavity, from accurate numerical solutions», Int. J. Heat Mass Trans., vol. 43, pp. 4043-4056, 2000.

[8] S. Xin, P. Le Quéré, «Linear stability analyses of natural convection flows in a differentially heated square cavity with conducting horizontal walls», Phys. Fluids, vol. 13, pp. 2529-2542, 2001.

[9] S. Xin, P. Le Quéré, "An extended Chebyshev pseudo-spectral benchmark for the 8:1 differentially heated cavity», Int. J. Numer. Meth. Fluids, vol. 40, pp. 981-998, 2002. 
[10] S. Xin, P. Le Quéré, "Natural-convection flows in air-filled, differentially heated cavities with adiabatic horizontal walls, Numer. Heat Transfer A», vol. 50, pp. 437-466, 2006.

[11] S. Xin, P. Le Quéré, "Stability of two-dimensional (2D) natural convection flows in air-filled differentially heated cavities: 2D/3D disturbances», Fluid Dynamics Research, vol. 44, p. 031419, 2012.

[12] Y. Zhao, C. Lei, J. C. Patterson, "Resonance of the thermal boundary layer adjacent to an isothermally heated vertical surface», J. Fluid Mech, vol. 724, pp. 305-336, 2013.

[13] L. E. Howle, «Control of Rayleigh-Benard convection in a small aspect ratio container», Int. J. Heat Mass Trans., vol. 40, pp. 817-822, 1997.

[14] L. E. Howle, "Linear stability analysis of controlled Rayleigh-Benard convection using shadowgraphic measurement», Phys. Fluids, vol. 9, pp. 3111-3113, 1997.

[15] B. Abourida, M. Hasnaoui, S. Douamna, «Transient natural convection in a square enclosure with horizontal walls submitted to periodic temperatures», Numerical Heat Transfer, Part A: Applications, vol. 36, pp. 737-750, 1999.

[16] S. Douamna, M. Hasnaoui, B. Abourida, «Two-dimensional transient natural convection in a repetitive geometry submitted to variable heating from below: Numerical identification of routes leading to chaos», Numer. Heat Transfer A, vol. 37, pp. 779-799, 2000.

[17] M. Z. Hossain, J. M. Floryan, «Natural convection in a fluid layer periodically heated from above», Phys. Rev. E, vol. 90, p. 023015, 2014.

[18] M. Z. Hossain, J. M. Floryan, «Natural convection in a horizontal fluid layer periodically heated from above and below», Phys. Rev. E, vol. 92, p. 023015, 2015.

[19] A. Nag, A. Sarkar, V. M. K. Sastri, «Natural-convection in a differentially heated square cavity with a horizontal partition plate on the hot-wall», Comput methods Appl Mech Eng, vol. 110, pp. 143156, 1993.

[20] E. Bilgen, "Natural convection in cavities with a thin fin on the hot wall», Int. J. Heat Mass Trans., vol. 48, pp. 3493-3505, 2005.

[21] F. Xu, J. C. Patterson, C. Lei, «Transition to a periodic flow induced by a thin fin on the sidewall of a differentially heated cavity», Int. J. Heat Mass Trans., vol. 52, pp. 620-628, 2009

[22] F. Xu, J. C. Patterson, C. Lei, «Effect of the fin length on natural convection flow transition in a cavity», Int J Therm Sci, vol. 70, pp. 92-101, 2013

[23] A. Ben-Nakhi, A. J. Chamkha, «Effect of length and inclination of a thin fin on natural convection in a square enclosure», Numer Heat Transf A, vol. 50, pp. 381-399, 2006.

[24] A. Ben-Nakhi, A. J. Chamkha, "Conjugate natural convection in a square enclosure with inclined thin fin of arbitrary length», Int J Therm Sci, vol. 46, pp. 467-478, 2007.

[25] M. Ghalambaz, E. Jamesahar, M. A. Ismael, A. J. Chamkha, «Fluid-structure interaction study of natural convection heat transfer over a flexible oscillating fin in a square cavity", Int J Therm Sci, vol. 111, pp. 256-273, 2017. 
[26] H. S. Kwak, J. M. Hyun, «Natural convection in an enclosure having a vertical sidewall with timevarying temperature», J. Fluid Mech, vol. 329, pp. 65-88, 1996.

[27] H. S. Kwak, K. Kuwahara, J. M. Hyun, «Resonant enhancement of natural convection heat transfer in a square enclosure», Int. J. Heat Mass Trans., vol. 41, pp. 2837-2846, 1998.

[28] P. S. Mahapatra, N. K. Manna, K. Ghosh, A. Mukhopadhyay, «Heat transfer assessment of an alternately active bi-heater undergoing transient natural convection», Int. J. Heat Mass Trans., vol. 83, pp. 450-464, 2015.

[29] P. S. Mahapatra, S. Chatterjee, A. Mukhopadhyay, N. K. Manna, K. Ghosh, «Proper orthogonal decomposition of thermally-induced flow structure in an enclosure with alternately active localized heat sources», Int. J. Heat Mass Trans., vol. 94, pp. 373-379, 2016.

[30] F. Penot, O. Skurtys, D. Saury, «Preliminary experiments on the control of natural convection in differentially-heated cavities», Int. J. Thermal Sciences, vol. 49, pp. 1911-1919, 2010.

[31] EDF, Code_Saturne, [Online]. Available: http://codesaturne.org/cms/sites/default/files/docs/4.0/user.pdf.

[32] J. C. Grondin, B. Roux, «Recherche de corrélations simples exprimant les pertes convectives dans une cavité bidimensionnelle, inclinée, chauffée différentiellement», Revue de Phy. App., p. 49, 1979.

[33] E. Gadoin, P. Le Quere, O. Daube, «A general methodology for investigating flow instabilities in complex geometries: application to natural convection in enclosures», Int. J. Numer. Meth. Fluids, vol. 37, pp. 175-208, 2001. 


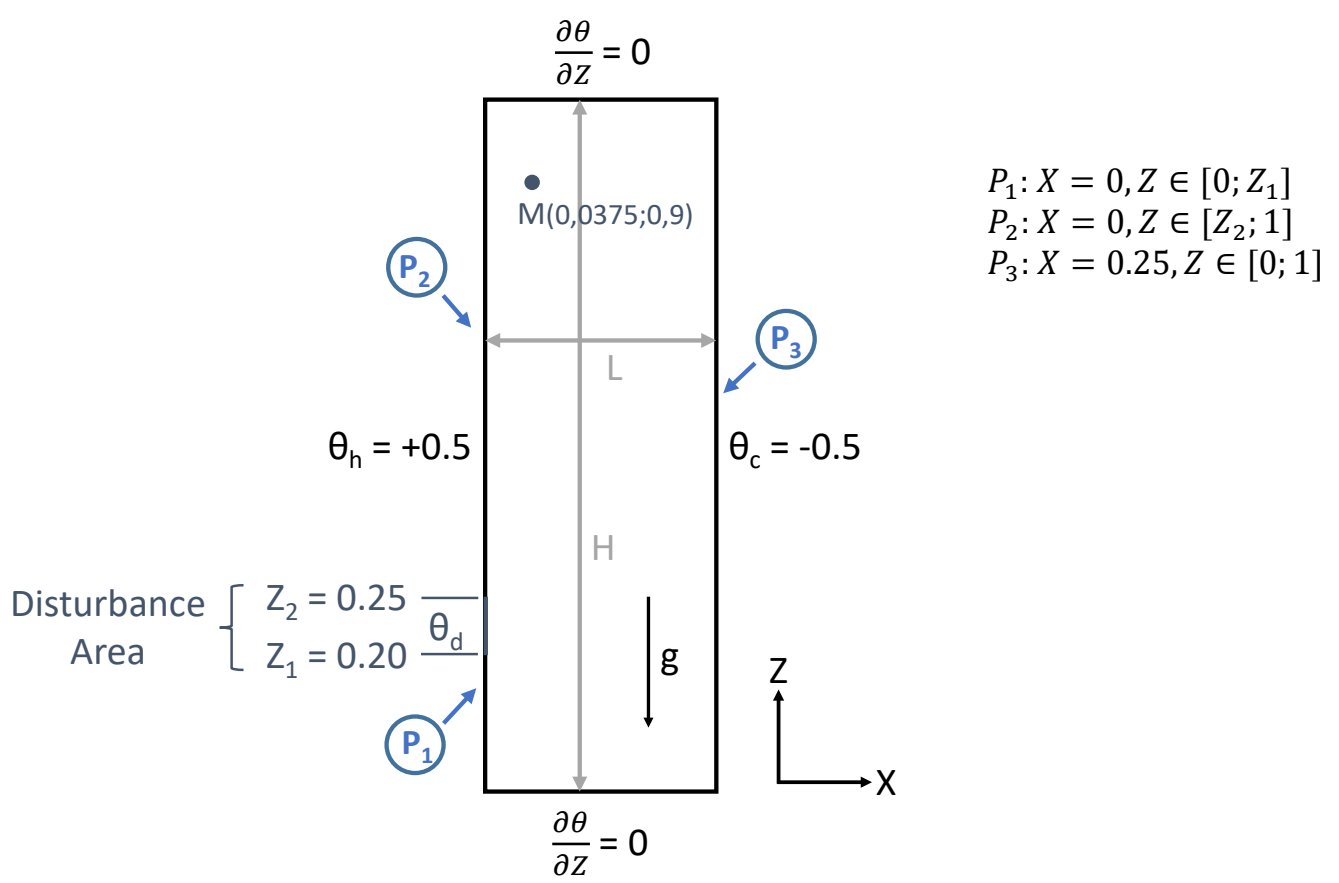

Fig. 1 : Scheme of the rectangular cavity with disturbance area on the hot wall for $Z$ between $Z_{1}$ and $Z_{2}$; the measuring point $M$ is located at $(X=0.0375 ; Z=0.9)$; the wall parts $\left(P_{1}, P_{2}, P_{3}\right)$ are respectively upstream and downstream the disturbance area, and all along the cold wall

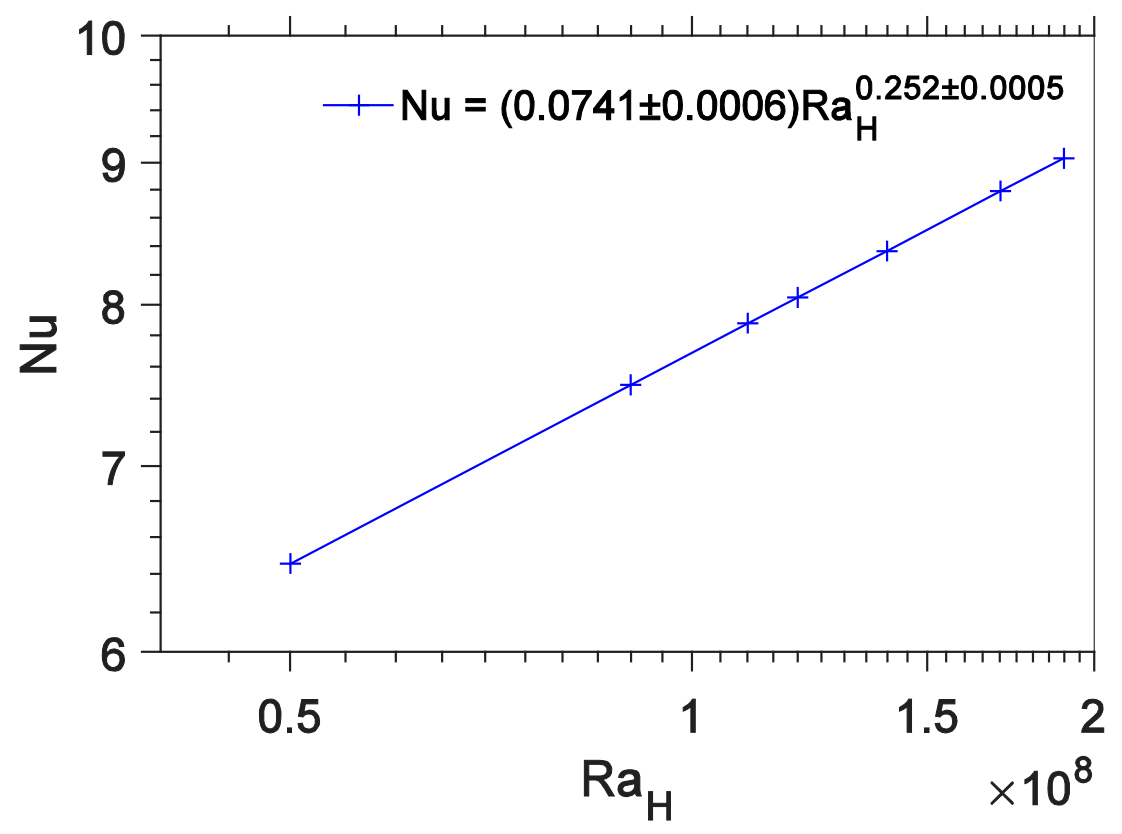

Fig. 2 : Evolution of the global (time-and space-averaged) Nusselt number with the Rayleigh number of the studied cavity; axis are displayed in a logarithmic scale 


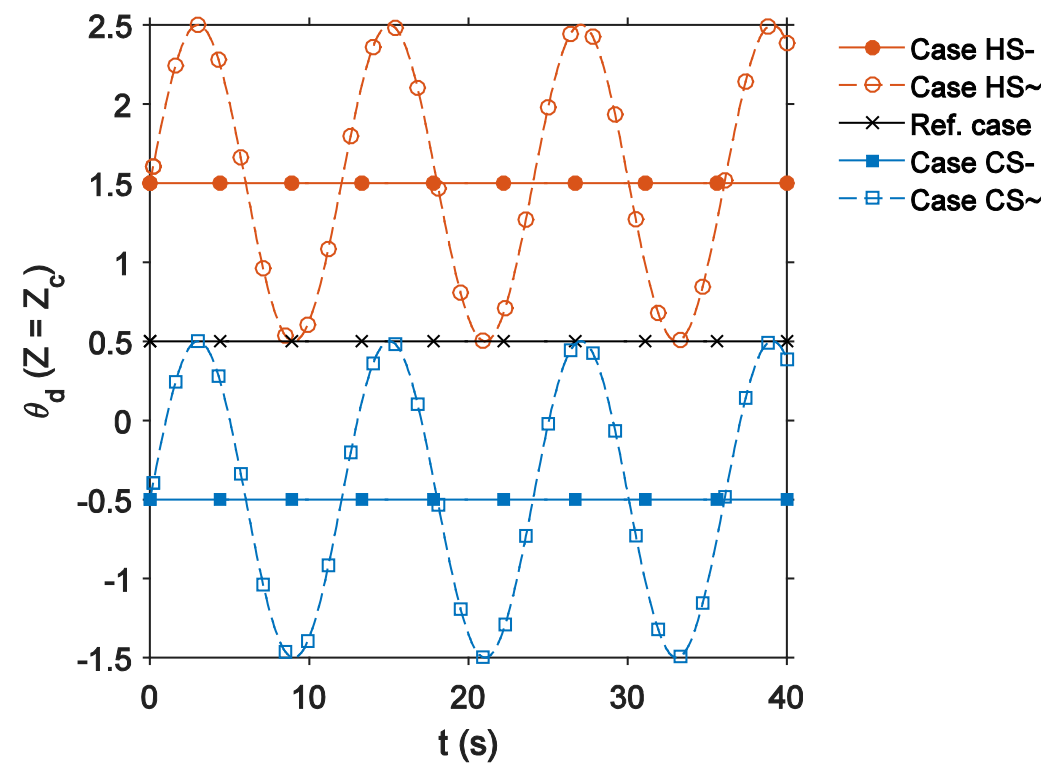

Fig. 3 : Temporal evolution of the temperature disturbance at the center of the disturbance area $\theta_{d}\left(Z=Z_{\text {cent }}\right)$ for all cases
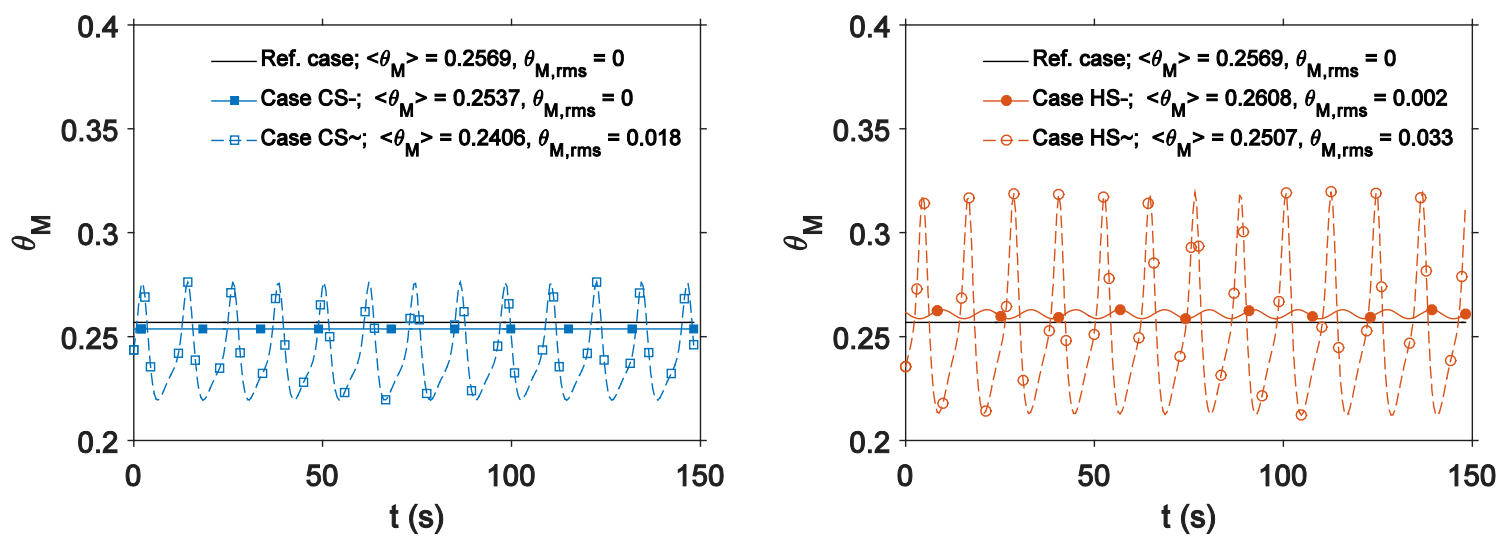

Fig. 4 : Time-evolution of the temperature plot at the measuring point M (see Fig. 1); left: ref. case, CS-and CS ; right: ref. case, HS- and HS 


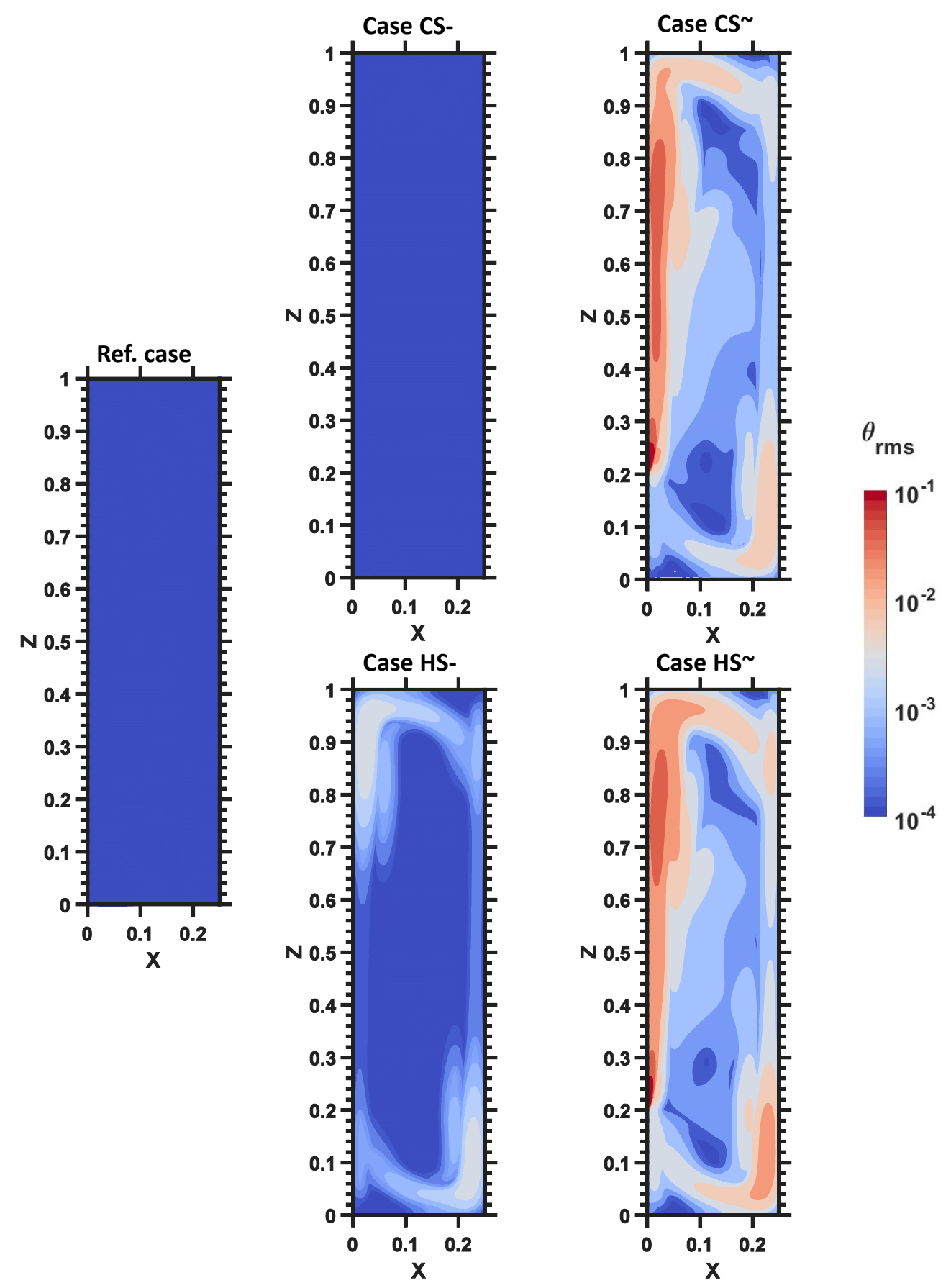

Fig. $5: \theta_{\text {rms }}$ in the whole cavity; top-left: ref. case, middle-top: case CS-, top-right: case CS , bottom-middle: case HS-, bottom-right: case HS 

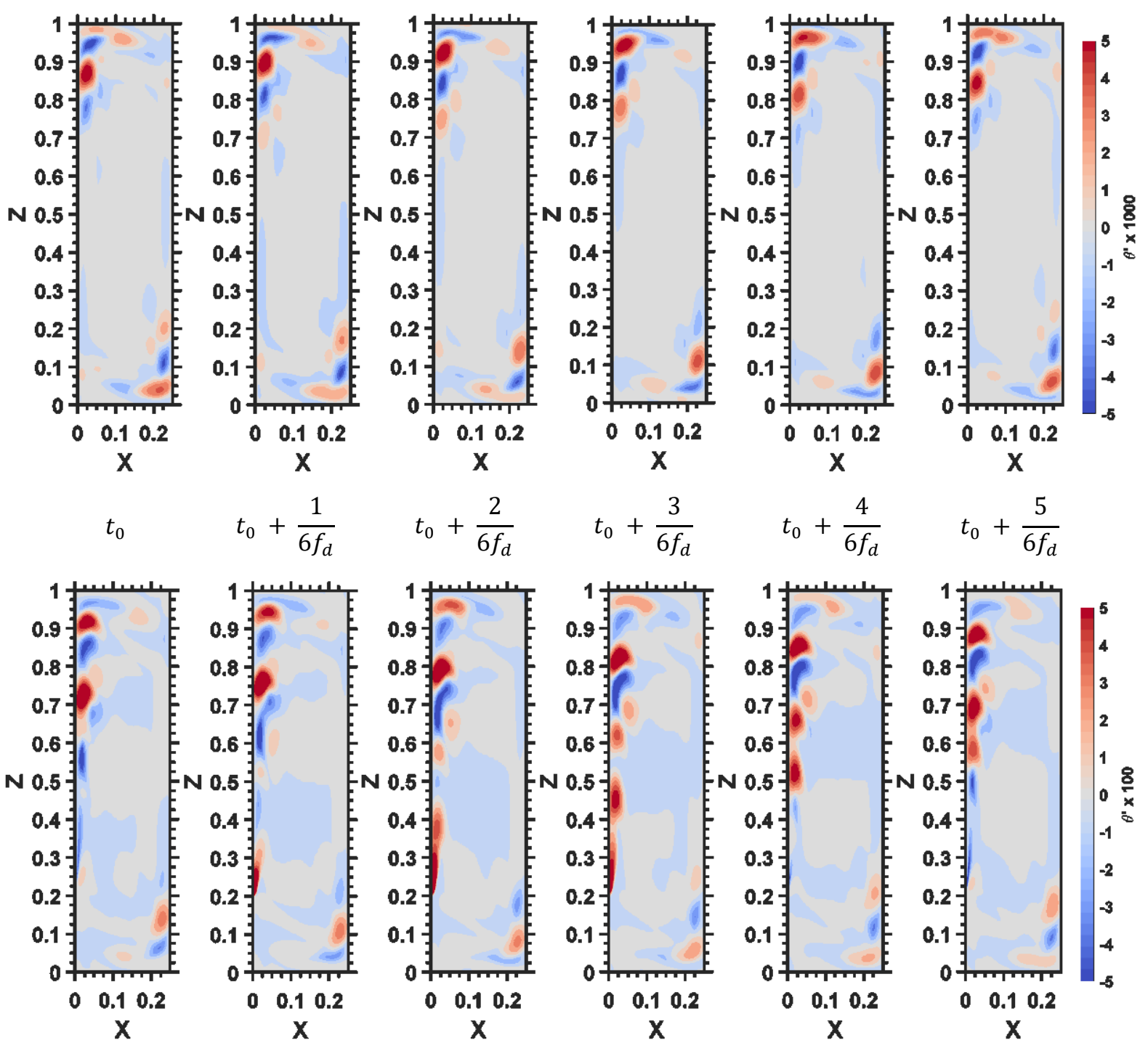

$$
t_{0}+\frac{1}{6 f_{d}}
$$

$t_{0}+\frac{2}{6 f_{d}}$

$t_{0}+\frac{3}{6 f_{d}}$

$t_{0}+\frac{4}{6 f_{d}}$

$t_{0}+\frac{5}{6 f_{d}}$
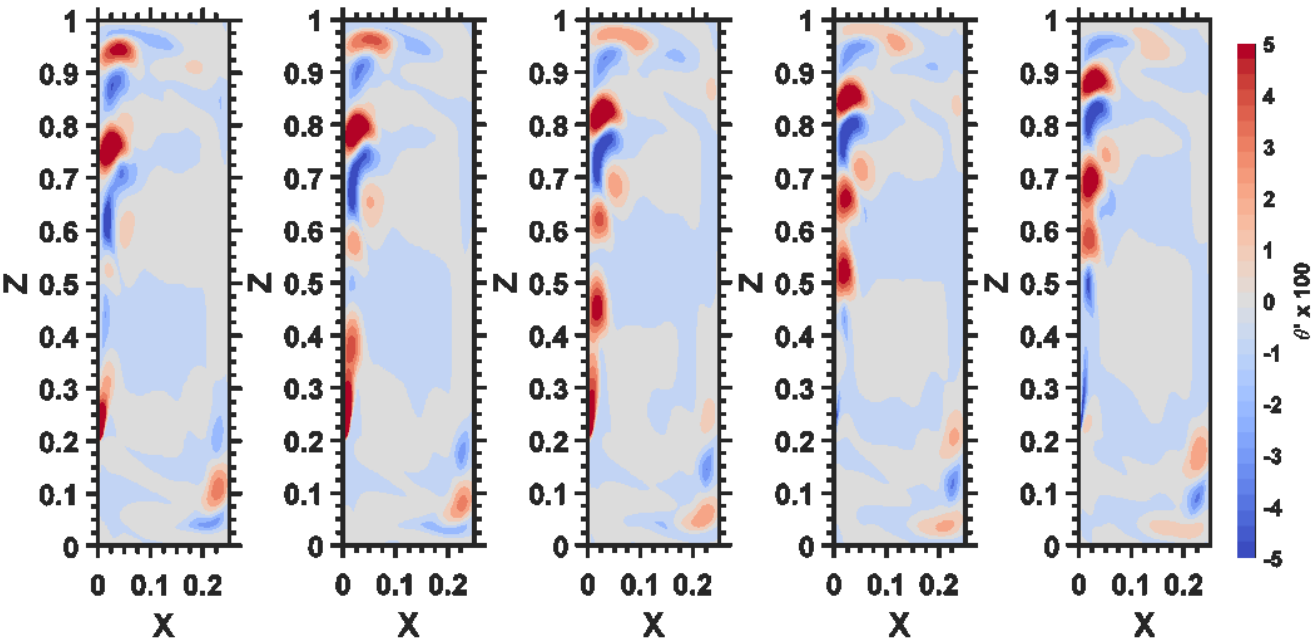

Fig. 6 : Temporal evolution of the temperature fluctuations $\theta^{\prime}=\theta-\langle\theta\rangle$ during one disturbance period $1 / f_{d}=2.5$; top: case HS-, bottom: case HS ; note that the scale for case HS is ten times the scale for case HS- 


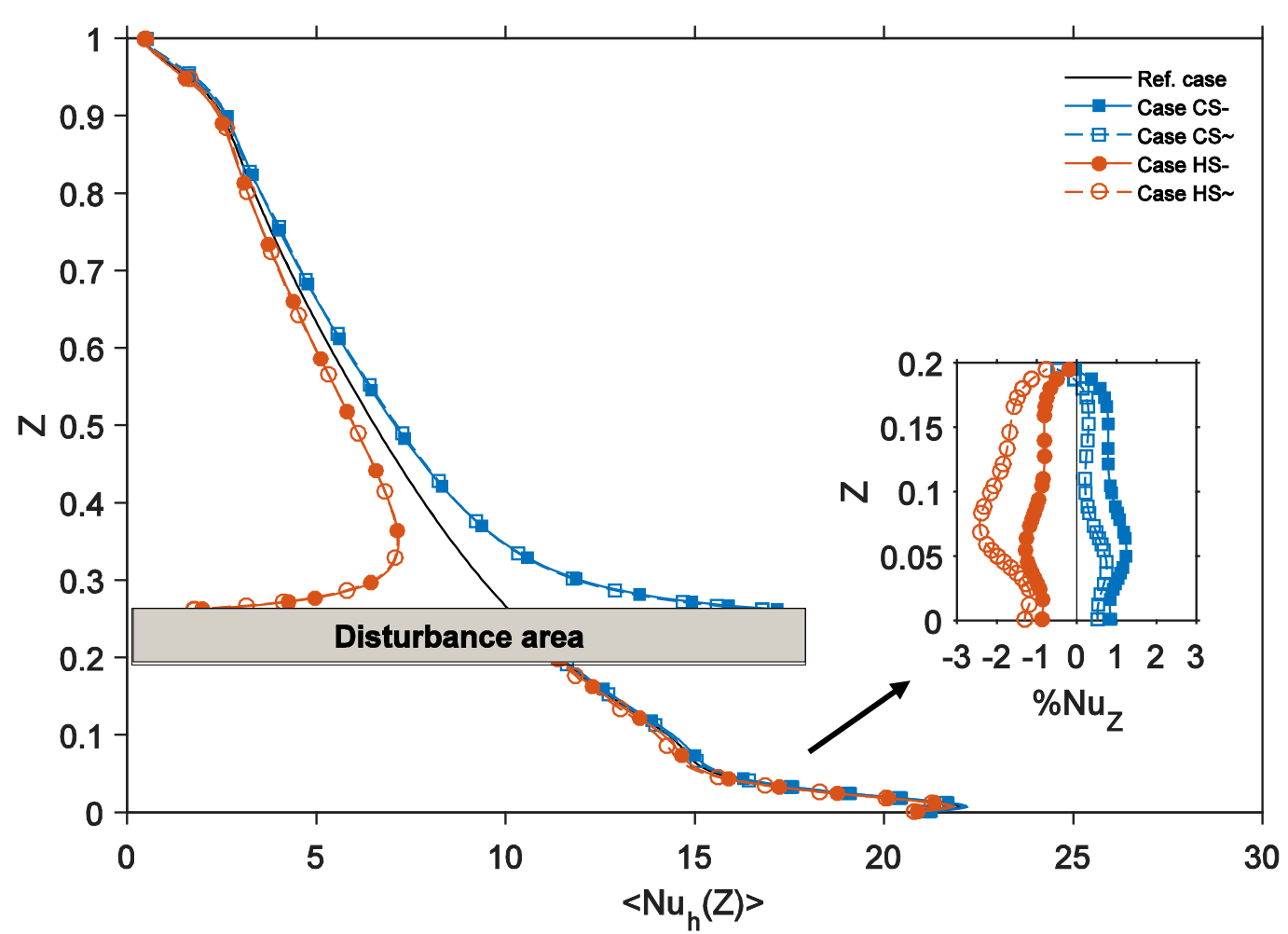

Fig. 7 : Local time-averaged Nusselt number $<N u_{h}(Z)>$ on the hot wall for all cases, and superimposed graph for local gain or loss respect to ref. case, $\% N u_{Z}=\frac{N u_{Z}-N u_{Z}(\text { ref.case })}{N u_{Z}(\text { ref.case })}$

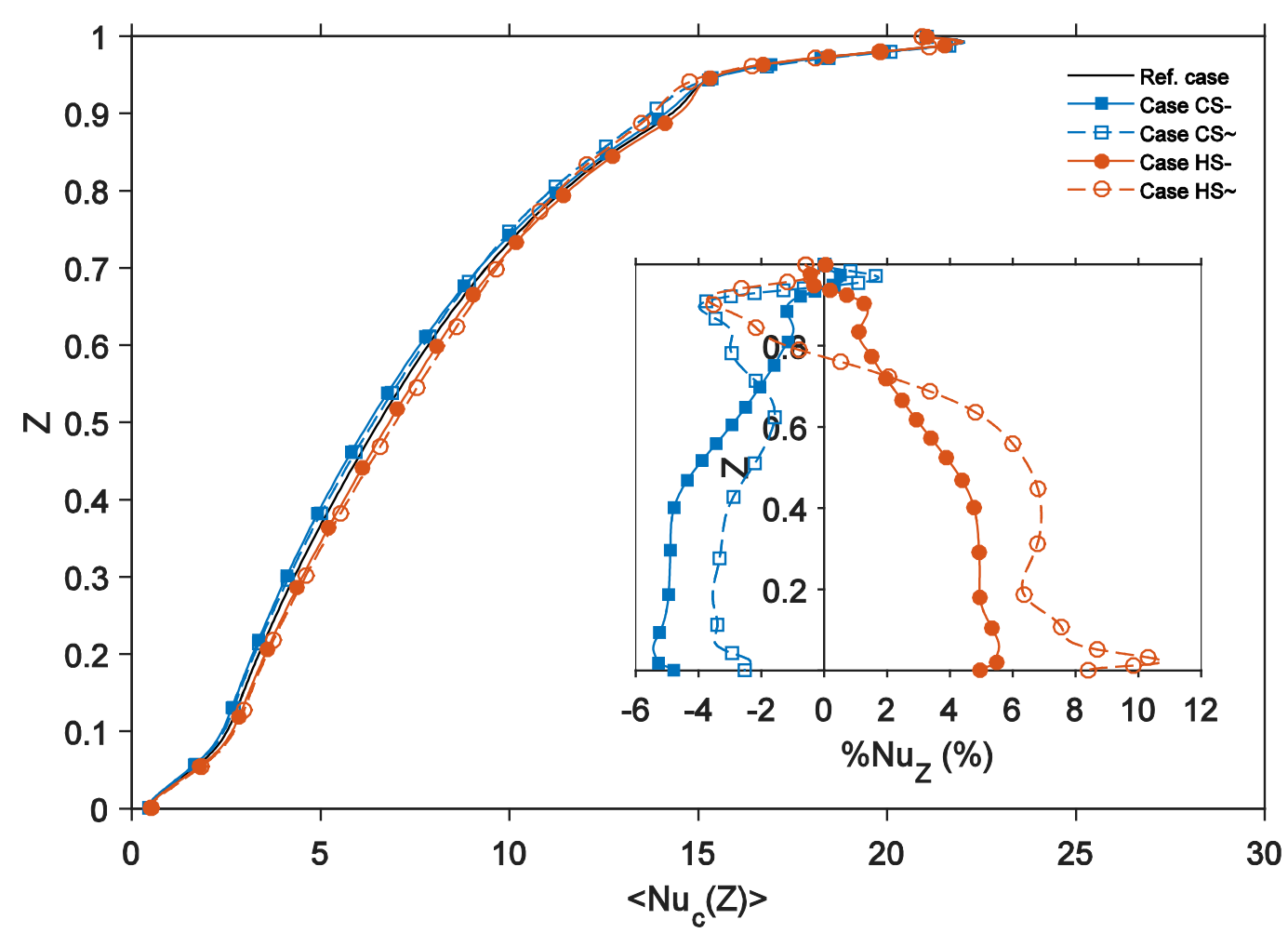

Fig. 8: Local time-averaged Nusselt number $<N u_{c}(Z)>$ at the cold wall for all cases, and superimposed graph for local gain or loss respect to ref. case, $\% N u_{Z}=\frac{<N u_{Z}>-N u_{Z}(\text { ref.case })}{N u_{Z}(\text { ref.case })}$ 


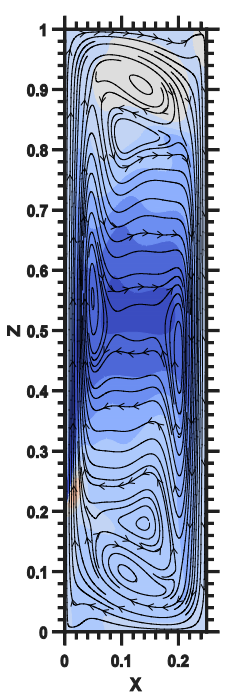

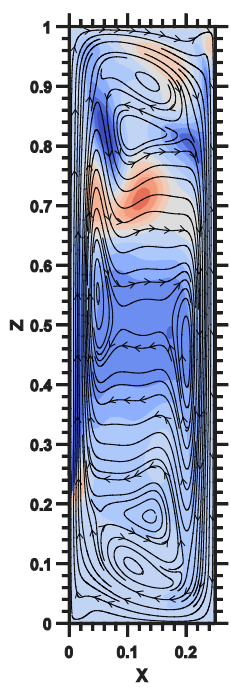

Case CS
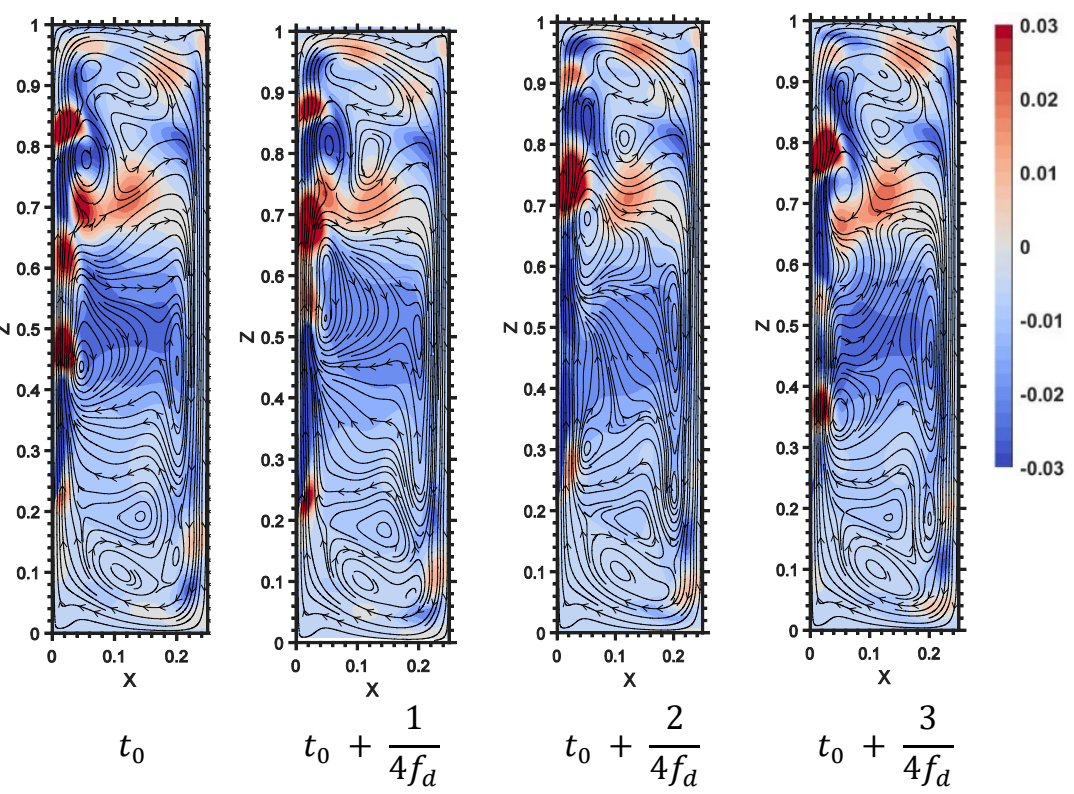

Fig.9: Left-hand two images: time-averaged values of relative temperature with respect to ref. case $\left\langle\theta-\theta_{\text {ref. }}\right.$ case $\rangle$ in cases CS- and CS ; right-hand four images: temporal evolution of instantaneous relative temperature with respect to ref. case $\theta-\theta_{\text {ref. case }}$ in case $C S^{\sim}$ during one disturbance period; streamlines are overprinted 

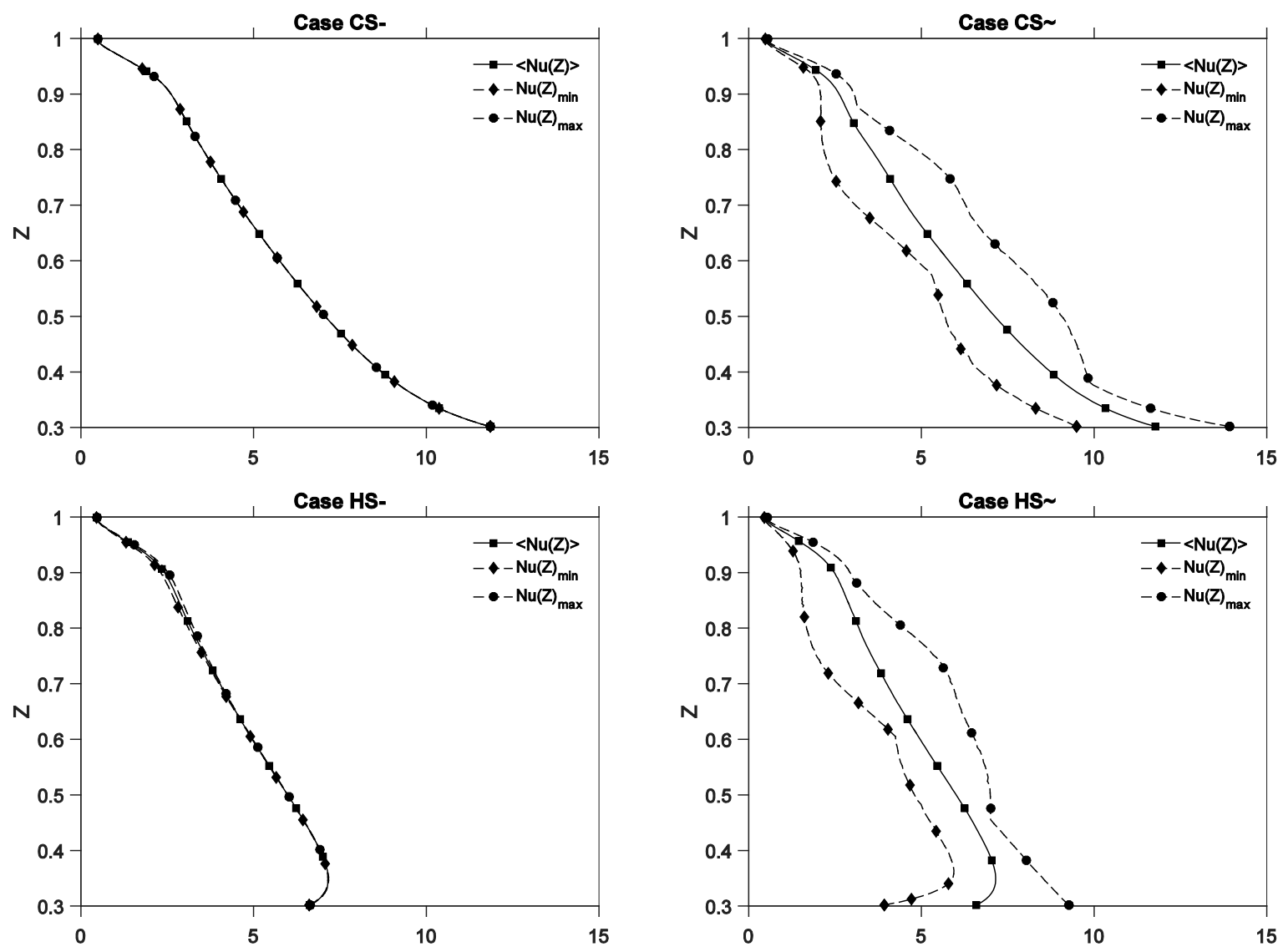

Fig. 10 : Profiles of time-averaged Nusselt number $\left\langle\mathrm{Nu}(\mathrm{Z})>\right.$ on the part $P_{2}$, bounded by its minimal and maximal values $\mathrm{Nu}(\mathrm{Z})_{\min }$ and $\mathrm{Nu}(\mathrm{Z})_{\max }$.

top-left: case CS-, top-right: case CS ; bottom-left: case HS-, bottom-right: case HS 


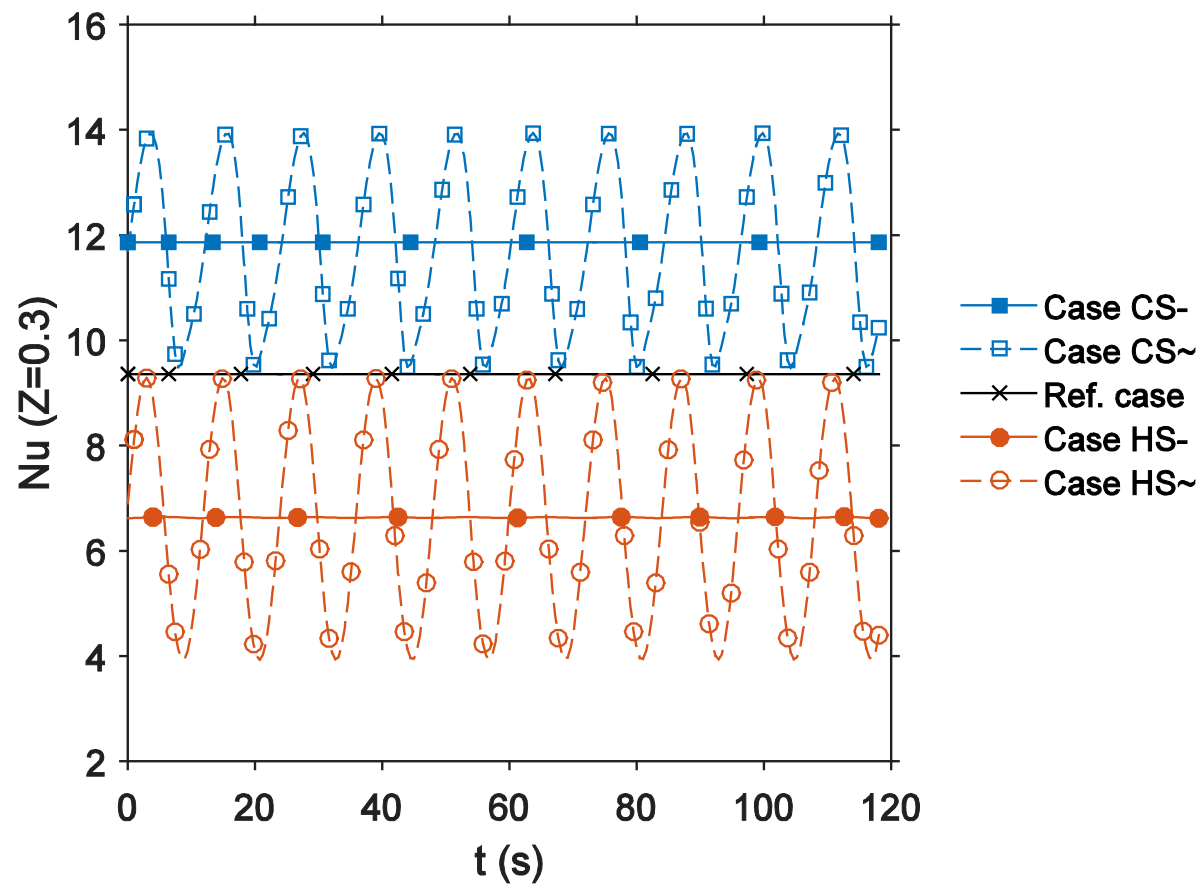

Fig. 11: Time evolution of local Nusselt number at $Z=0.3$ on the hot wall, for the 5 investigated cases

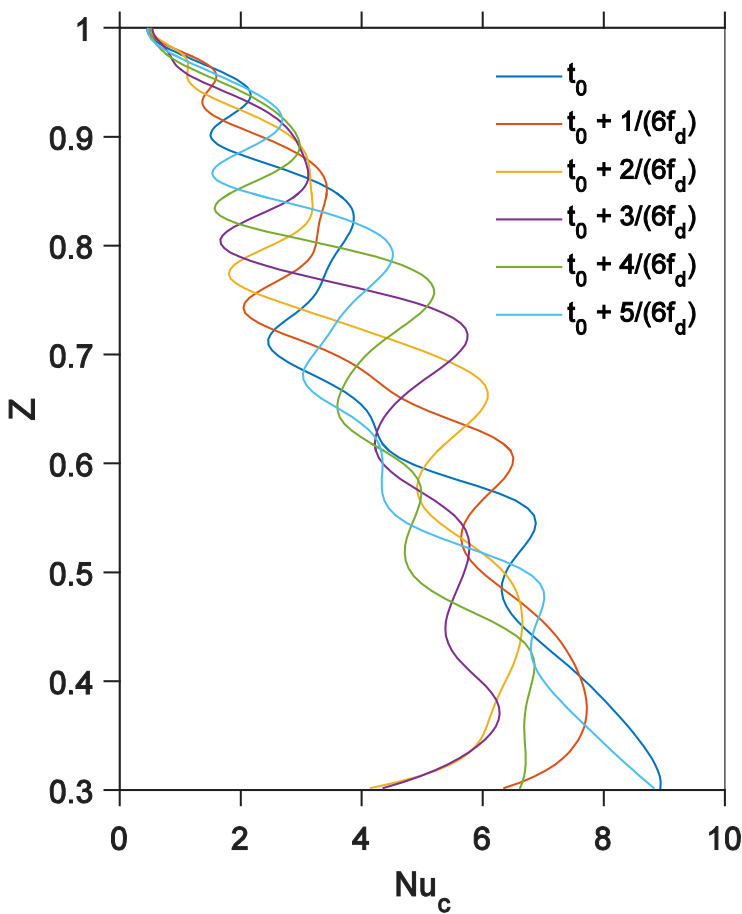

Fig. 12 : Snapshots of local Nusselt numbers at the hot wall for 6 times equally distributed over a period. $H S^{\sim}$ case, $Z \in[0.3 ; 1]$. 


\begin{tabular}{ccccc}
\hline & \multicolumn{2}{c}{$f_{\text {crit }}$} & \multicolumn{2}{c}{$\operatorname{Ra}_{H, \text { crit }}\left(\mathrm{x} 10^{-8}\right)$} \\
Branch & Present & Xin [10] & Present & Xin [10] \\
\hline 1 & 0.403 & 0.404 & 1.052 & 1.032 \\
2 & 0.434 & 0.433 & 1.050 & 1.056 \\
3 & 0.467 & 0.468 & 1.180 & 1.183 \\
4 & 0.506 & 0.507 & 1.273 & 1.291 \\
5 & 0.545 & 0.545 & 1.403 & 1.421 \\
\hline
\end{tabular}

Table 1: Dimensionless critical frequencies $f_{\text {crit }}$ and critical Rayleigh numbers $R a_{H, \text { crit }}$ for the five first branches; Comparison with benchmark solutions from Xin [10].

\begin{tabular}{cccccc}
\hline & Ref. case & Case CS- & Case CS & Case HS- & Case HS \\
\hline $\mathrm{A}_{\mathrm{d}}$ & 0 & 1 & 1 & 1 & 1 \\
$\delta$ & - & -1 & -1 & 1 & 1 \\
$\varepsilon$ & - & 0 & 1 & 0 & 1 \\
$\mathrm{f}_{\mathrm{d}}$ & - & - & 0.40 & - & 0.40 \\
\hline
\end{tabular}

Table 2: Studied cases; CS and HS denote cases with respectively a lower $(\delta=-1)$ and a higher $(\delta=+1)$ disturbance temperature than the hot wall temperature ('cold spot' and 'hot spot'); symbols '- ' and ' ' denote cases with respectively a constant $(\varepsilon=0)$ and a sine $(\varepsilon=1)$ disturbance

\begin{tabular}{lccccc}
\hline & Ref. case & Case CS- & Case CS & Case HS- & Case HS \\
\cline { 2 - 6 } & 14.825 & $14.954(+0.9 \%)$ & $14.881(+0.4 \%)$ & $14.690(-0.9 \%)$ & $14.573(-1.7 \%)$ \\
$<\overline{N u_{P_{1}}}>$ & 5.326 & $6.169(+15.8 \%)$ & $6.166(+15.8 \%)$ & $4.409(-17.2 \%)$ & $4.407(-17.3 \%)$ \\
$<\overline{N u_{P_{2}}}>$ & 7.486 & $7.317(-2.3 \%)$ & $7.321(-2.2 \%)$ & $7.662(+2.4 \%)$ & $7.648(+2.2 \%)$ \\
\hline$\overline{N u_{P_{3}}}>$ & $7.3 \%)$ &
\end{tabular}

Table 3: Values of zonal Nusselt numbers on wall parts upstream disturbance area $\left(P_{1}\right)$, downstream the disturbance area $\left(P_{2}\right)$ and at the cold wall $\left(P_{3}\right)$ (see Fig. 1). Comparison with the ref. case. 